\title{
Due o tre cose che (non) sappiamo di lei. L'autoepitaffio della Pia
}

Deux ou trois choses que l'on (ne) sait pas d'elle. L'auto-épitaphe de Pia

Two or Three Things We (Don't) Know about Her. Pia's Self-Epitaph

\section{Francesco Bausi}

\section{(2) OpenEdition}

\section{Journals}

Edizione digitale

URL: https://journals.openedition.org/cei/9429

DOI: $10.4000 /$ cei.9429

ISSN: 2260-779X

\section{Editore}

UGA Éditions/Université Grenoble Alpes

\section{Edizione cartacea}

ISBN: 978-2-37747-304-5

ISSN: $1770-9571$

\section{Notizia bibliografica digitale}

Francesco Bausi, «Due o tre cose che (non) sappiamo di lei. L'autoepitaffio della Pia», Cahiers d'études italiennes [Online], 33 | 2021, online dal 01 octobre 2021, consultato il 13 octobre 2021. URL: http:// journals.openedition.org/cei/9429; DOI: https://doi.org/10.4000/cei.9429

Questo documento è stato generato automaticamente il 13 octobre 2021 


\title{
Due o tre cose che (non) sappiamo di lei. L'autoepitaffio della Pia
}

\author{
Deux ou trois choses que l'on (ne) sait pas d'elle. L'auto-épitaphe de Pia \\ Two or Three Things We (Don't) Know about Her. Pia's Self-Epitaph
}

\section{Francesco Bausi}

«Fa sempre freddo in quelle mura, il cielo è chiaro ma la terra resta scura».

Gianna Nannini, Dolente Pia

1 La moderna esegesi della Commedia ha quasi unanimemente applicato sui pochi versi dedicati in conclusione del quinto del Purgatorio all'enigmatica figura della Pia (appena sette, dei quali sei da lei pronunciati) due tenaci etichette, ormai entrate nella vulgata critica e riassumibili nelle nozioni di reticenza e di elegia. Da una parte, infatti, si sostiene che la donna, con pudico riserbo, fornisca intenzionalmente su di sé poche e vaghe informazioni, e che dunque il poeta abbia voluto avvolgere il personaggio e la sua vicenda in un alone di indeterminatezza (che gli interpreti dovrebbero avere la sensibilità e il buon gusto di non violare); dall'altra, nessuno o quasi si esime dal sottolineare la dolcezza gentile del personaggio, che si rifletterebbe sul tono mestamente elegiaco e sulla tenue musicalità di questi celebri versi. Nozioni ed etichette a mio avviso non del tutto veritiere, e responsabili anzi di un approccio semplicistico e di una lettura monocorde che rischiano di banalizzare il senso e la forza dell'episodio, oltre che di condizionare persino le non irrilevanti decisioni ecdotiche cui la tradizione obbliga gli editori nell'ultimo verso del canto.

2 L'episodio, infatti, accumula nel suo brevissimo spazio due ordini di problemi, complicando quelli derivanti dalla nostra ignoranza dei fatti e dalla conseguente impossibilità di identificare con sicurezza i personaggi con quelli sollevati dal serio dubbio testuale che interessa il v. 136: dubbio che tutto lascia credere generato nei copisti proprio dalla difficoltà di comprendere i due versi finali e l'intera vicenda della Pia. Storia e filologia, insomma, congiurano nel serrare in un nodo di incertezza pressoché inestricabile la chiusa del canto: causa non ultima della irresistibile attrattiva che essa ha esercitato sui lettori di ogni epoca. 


\section{La reticenza}

Di sé, la donna sembra dire solo quattro cose: che ha nome Pia; che è nata a Siena e morta in Maremma; che è morta di morte violenta (dato, questo, già deducibile senza ombra di dubbio dalla sua appartenenza alla terza schiera degli spiriti dell'antipurgatorio); che nella sua morte fu in qualche modo coinvolto il marito. Non è questo l'unico caso di un personaggio non per fama noto sul quale il poeta sia avaro di notizie biografiche: altrettanto poco ci dice, ad esempio, del Navarrese (Inf. XXII, dove resta ignoto anche il suo nome, supplito, non si sa con quanta attendibilità, dai primi commentatori) ${ }^{1}$ o di Marco Lombardo (Purg. XVI), che pure rivestono, nell'economia narrativa dei rispettivi canti, un ruolo ben altrimenti rilevante. Se qui i silenzi danteschi colpiscono particolarmente e accendono il desiderio di saperne di più, ciò dipende - oltre che, ovviamente, dal fascino del personaggio - anche dal contrasto tra l'episodio della Pia e i due che lo precedono nello stesso canto, dove Iacopo del Cassero e Bonconte da Montefeltro si dilungano nel racconto della propria morte, precisandone modi e circostanze con quasi eccessiva e ossessiva attenzione ai dettagli. Il poeta obbedisce innanzitutto a quel principio di varietas che è uno dei criteri compositivi fondamentali della sua opera: come a due uomini segue, in fine di canto, una donna, così l'ultimo quadro si contraddistingue per una concisa brevitas che fa da contraltare agli effusi monologhi di Iacopo (21 versi) e ancor più di Bonconte (45 versi). Brevitas suggerita anche dalla natura della violenza subita dalla Pia. Se infatti - come tre sono le forme di superbia, quelle della stirpe, dell'intelletto e del potere, punite nell'ordine, nell'XI del Purgatorio, in Omberto Aldobrandeschi, Oderisi da Gubbio e Provenzan Salvani - il canto V esemplifica nei suoi protagonisti tre generi di violenza (politica, militare e privata), è naturale che nella sfera del privato Dante eviti di addentrarsi; non diversamente si era comportato con Paolo e Francesca, tacendo delle modalità del loro assassinio, e limitandosi a informare, per bocca della Polentana, che i due furono uccisi a tradimento da un parente, destinato per questo alla Caina (v.107), e che si indovina essere stato il marito di lei. Da questo dipende, nel finale del V del Purgatorio, anche la variatio tonale da molti osservata, che, pur nella persistenza del basso continuo tragico (come si addice al canto dei morti per forza), fa seguire, alla grandiosa e quasi apocalittica scena evocata da Bonconte, una chiusa stringata ed epigrammatica, quasi in dissolvenza.

4 Non necessariamente, dunque, la reticenza dantesca deriverà - come talora si afferma - dal fatto che, in casi del genere, persone e eventi fossero di dominio pubblico, o comunque noti alla maggioranza dei lettori: cosa che, oltre a essere difficile da ammettere, può forse avere un fondamento per Paolo e Francesca, non certo per la Pia, chiunque ella fosse e comunque sia stata uccisa. Poteva davvero Dante, anche - per riprendere un esempio appena addotto - introducendo nel XXII dell'Inferno il Navarrese (vv. 48-54), che pure esaurisce in pochissimi versi (cinque) un suo scarno profilo biografico, credere che il lettore avrebbe potuto identificare questi personaggi? Se la cosa non fosse difficilmente ammissibile per la Commedia, potremmo addirittura pensare che si tratti di personaggi d'invenzione (dove mai l'Alighieri può aver appreso notizie sulla vita dell'oscuro barattiere di Navarra? Benvenuto deve addirittura immaginare che il poeta lo abbia fatto durante il suo soggiorno francese ${ }^{2}$; ma se i personaggi non sono mai di fantasia, inventate sono non di rado certe circostanze della 
loro esistenza, come, per rimanere in questo canto, i pentimenti in articulo mortis di Iacopo, di Bonconte e della stessa Pia, intorno ai quali non potevano sussistere testimonianze ${ }^{3}$.

5 Le due spiegazioni suggerite da Giorgio Inglese («in questo caso, come in altri, Dante sopravvalutò forse la fama dell'episodio; o, più semplicemente, ritenne superfluo ogni dettaglio, rispetto all'evidenza poetica della Pia») $)^{4}$ sono sensate e possono essere condivise (e un'altra se ne può aggiungere, chiamando in causa la frammentarietà e l'incertezza delle notizie che lo stesso Dante doveva aver raccolto in merito alla donna e alla vicenda durante uno dei suoi soggiorni senesi), ma non risolvono ogni difficoltà. Quanto alla seconda, è indubbio che in un episodio di questo genere l'«evidenza poetica» (e, direi inoltre, 'esemplare': esemplare, intendo, anche di uno specifico tipo di morte violenta) sia ciò che più conta. Né può contestarsi, in linea di principio, che Dante dica sempre ciò che al lettore è necessario sapere: constatazione, questa, che può spiacere a certi dantisti, ma che è inconfutabile e che sempre dovremmo tenere a mente, se non vogliamo ridurre il poema a un gioco enigmistico o a una palestra di erudizione ${ }^{5}$. Nondimeno, se è generalmente salutare l'esortazione a star contenti al quia del testo, evitando spericolate introspezioni psicologiche, malcerte ricostruzioni storiche, capziosi e arbitrari collegamenti intertestuali o astratte analisi formalistiche, suona talora stucchevole continuare a insistere sull'inopportunità dei tentativi di penetrare nel 'non detto', attenendosi - nel caso specifico - a una condotta sobria e prudente, che non faccia violenza al pudore del poeta e del personaggio.

6 Anche prescindendo dal fatto che spesso, come si sa, nella Commedia sono lasciati volutamente impliciti dati e concetti - talora anche rilevanti - che spetta al lettore ricavare per induzione dall'attenta analisi del testo, tale invito rischia, soprattutto in un episodio come quello della Pia, di autorizzare comode scorciatoie esegetiche che perpetuano letture ripetitive e che in ultima analisi incoraggiano la superficiale 'degustazione' impressionistica della poesia. Lecito e anzi doveroso è pertanto lo sforzo di estrarre dal dettato dantesco - beninteso, senza eccessi di sottiglianza - ciò che in esso è implicitamente contenuto, vale a dire legittimamente deducibile da quei versi o da altri luoghi del poema con i quali il passo in esame intrattenga rapporti diretti e non puramente esterni (come quelli che taluni ritengono di poter ricavare da fenomeni di intratestualità 'debole': la ricorrenza di singoli lemmi, ad esempio, o la ripetizione di certe rime). C'è differenza, insomma, tra non indugiare su irrilevanti dettagli storicobiografici e concentrare allusivamente in pochi versi il maggior numero possibile di informazioni su un avvenimento o su un personaggio: come è proprio dell'arte sommamente brachilogica di Dante ${ }^{6}$, e come, a mio avviso, il poeta ha voluto fare nel sintetico e criptico 'medaglione' della Pia. In altre e più precise parole: poiché la caratterizzazione dei personaggi, nella Commedia, si concentra esclusivamente sui momenti realmente 'decisivi' della loro vita, quelli che ne hanno segnato per sempre l'esistenza nella prospettiva del destino ultraterreno ${ }^{7}$, da un lato è vano e improprio, per la comprensione del poema e della funzione tipologica ed esemplare in esso svolta dai suoi personaggi, andare a caccia di notizie biografiche allotrie e inessenziali, ma dall'altro è opportuno e anzi necessario sforzarsi di ricavare dai pochi e stringati accenni cui spesso Dante si limita tutto quanto pertiene alla 'quintessenza' del personaggio e ne fissa, spiegandola, la sorte ultima: quegli avvenimenti o quei tratti fondamentali che esplicitamente o allusivamente vanno a costituire, per dirla ancora 
con Auerbach, quella «arcana cifra» cui il poeta riduce, con mirabile forza di astrazione, le molteplici vicende degli uomini ${ }^{8}$.

\section{La colpa}

7 Il cosiddetto 'antipurgatorio' (il termine, come si sa, non ricorre mai nella Commedia, al pari di 'antinferno') è una zona altamente problematica dell'oltretomba dantesco, giacché manca di veri e propri precedenti nella speculazione teologica e nella letteratura visionaria medievale, e perché Dante non si impegna né in una sua precisa definizione teologica, né in una sua accurata descrizione topografica. Molto rimane non detto o indeterminato, e i caratteri dell'antipurgatorio devono spesso essere ricostruiti in base ad accenni sparsi e a volte generici disseminati nel tessuto dei primi otto canti; donde i numerosi e non lievi dubbi - tanto di carattere strutturale, quanto in relazione a luoghi specifici e puntuali - sollevati da questa sezione iniziale della seconda cantica9.

8 Ad esempio, è evidente che se la 'negligenza' (che ritardò il pentimento dei peccati fino al momento estremo della vita) accomuna tutte le anime dell'antipurgatorio, la loro ripartizione in quattro schiere non obbedisce a un criterio univoco ${ }^{10}$ : mentre, infatti, la condizione delle anime appartenenti ai primi due gruppi e al quarto fu sancita da un atto ufficiale della Chiesa (la scomunica) o da una personale disposizione dell'animo (la pigrizia, nel senso più comune della parola; o l'eccessivo attaccamento al proprio ruolo terreno, che contraddistinse i principi negligenti), diverso è il presupposto sul quale è costituita la categoria dei morti di morte violenta, giacché quest'ultima è un accidente 'esterno', imprevedibile e fortuito, e tale quindi da imprimere il marchio della negligenza su anime che, se la vita avesse avuto il suo corso normale, avrebbero potuto evitare di incorrere in quella colpa. È il caso, per restare al V canto, di Bonconte, laddove suo padre Guido - prescindendo dal suo ultimo e fatale peccato - ebbe il tempo e il modo, in vita, di pentirsi e di fare «ammenda», diventando, da «uom d'arme», «cordigliero» (Inf. XXVII, 67). Ne consegue che, stante la gravità decrescente delle colpe nel purgatorio, i morti di morte violenta sono per Dante meno colpevoli non solo, com'è ovvio, degli scomunicati (che infatti si trovano ai piedi della montagna, e ancora non possono accedervi), ma anche dei pigri ${ }^{11}$; mentre i principi negligenti beneficiano di un trattamento di favore - teologicamente poco giustificato, a dire il vero, al pari di quello dei limbicoli, che con essi presentano non pochi tratti in comune - in grazia dell'importante funzione politica ad essi un tempo affidata nel mondo.

9 La 'negligenza' imputata alle anime dell'antipurgatorio non coincide con la negligentia tomistica (che è un peccato speciale, trattandosi di un difetto spirituale, ossia di un manchevole fervore di carità o della mancanza di carità tout court), ma corrisponde piuttosto a quella che Tommaso definisce pigritia e che semplicemente «importat tarditatem ad exequendum» (nel caso specifico, a pentirsi) ${ }^{12}$ : è dunque una «disposizione al peccato ${ }^{13}$, o, se si preferisce, un peccato supplementare, che, avendo ritardato colpevolmente la conversione fino all'ultimo momento della vita, richiede una pena e una espiazione apposite e specifiche, e che si aggiunge ai peccati commessi da ogni anima (che saranno poi scontati nelle rispettive cornici). Questa forma di negligenza viene punita nell'antipurgatorio, che pertanto non è un luogo di semplice 'attesa', ma di vera e propria pena (ossia, di pena in quanto di attesa, posto che gli spiriti purganti 
desiderano abbreviare il più possibile la permanenza nel loro stato, e che «perder tempo a chi più sa più spiace $»^{14}$ ), pur trattandosi solo, in effetti, di una pena 'preliminare', che esclude ogni forma di sofferenza fisica.

Potremmo così definire l'antipurgatorio una sorta di Limbo purgatoriale (e dunque 'temporaneo'), dal quale le anime passeranno poi alle cornici corrispondenti alle vere e proprie, specifiche colpe di cui si macchiarono. Il fatto che Dante non espliciti, per nessuna di queste anime, a quali delle sette balze purgatoriali esse saranno destinate (limitandosi a sottolineare, talora, la terribile gravità dei loro peccati: quelli «orribili» di Manfredi, o le «gravi offese» di Iacopo del Cassero), non obbliga a concludere - come solitamente si afferma - che sia impossibile dedurre quali furono tali colpe, e che il poeta non fornisca indizi utili in tal senso. Tutto lascia supporre che per uomini di potere e di guerra come Manfredi, Iacopo del Cassero e Bonconte si sarà trattato di superbia (che sarebbe in causa anche se Dante avesse considerato Manfredi un eretico) e di avarizia o cupidigia (intesa, in questo caso, come sete di potere), e almeno per gli ultimi due, probabilmente, anche di invidia e di ira; peccati analoghi dovranno essere invocati per i principi della valletta (a prescindere da altre colpe particolari, come l'accidia di Rodolfo imperatore, che ha «negletto ciò che far dovea» [VII, 92]); l'oscuro Belacqua presenta chiaramente i tratti dell'accidioso e forse anche quelli del goloso; a Sordello, sulla base delle notizie che circolavano su di lui e che Dante poteva ricavare anche da testimonianze letterarie, era automatico associare la lussuria (in virtù dei suoi tempestosi amori con Cunizza) ${ }^{15} \mathrm{e}$ forse anche - per il modo in cui è qui presentato: «anima [...] altera e disdegnosa», paragonata a un «leon quando si posa» (VI, 61-62 e 66) - la superbia (fu d'altronde potente barone e alto feudatario di Carlo I d'Angiò).

11 Per quanto riguarda la Pia, non si incorre in una gratuita speculazione se osserviamo che, in episodi di violenza matrimoniale, la colpa deve avere a che fare con la sfera privata, cioè con l'àmbito affettivo e relazionale; lo conferma il modo ellittico in cui la donna allude al marito: «Salsi colui...». Si è spesso sottolineata la delicatezza di questo accenno finale: ma, come si è detto, va evitato il rischio di enfatizzare in modo un po' dolciastro la tonalità 'elegiaca' dell'episodio. Pia non è una vittima innocente, e se il marito è colpevole, lo è stata - in altro modo, e benché pentitasi - anche lei ${ }^{16}$. Da qui, oltre che dal perdono e dalla pacificazione con cui (come tutti gli spiriti del purgatorio, e come i morti di morte violenta in particolare) è uscita dalla vita ${ }^{17}$, scaturisce quell'atteggiamento 'assolutorio' verso il consorte che molti interpreti hanno messo in evidenza, e che non si riscontra esplicitamente, nei confronti dei loro uccisori, in Iacopo (che giudica anzi eccessiva l'ira con cui Azzo VIII d'Este volle vendicarsi di lui) e in Bonconte.

12 Tuttavia, i due versi conclusivi non devono necessariamente leggersi solo in questa chiave. In quel «Salsi colui» può altrettanto legittimamente sentirsi, se non una sfumatura di risentimento (inammissibile in un'anima del purgatorio), almeno un'accusa nei confronti del marito ancora vivente, o per dir meglio un suo smascheramento e una ferma denuncia di colpevolezza ${ }^{18}$ : d'altronde, se fosse stato di dominio pubblico che Pia era stata uccisa dal marito o per volontà di lui, la formulazione degli ultimi due versi, e in particolare l'attacco del penultimo, risulterebbe immotivata e francamente assurda (il senso è infatti: «come e perché la Maremma mi disfece, lo sa bene colui che mi prese in moglie, e solo lui») ${ }^{19}$. Identico valore hanno questa espressione, e questa mossa sintattica, in Purg. XXXI, 89-90, in riferimento a Beatrice, che sola sa - avendo assistito alla scena, e avendo provocato il 
pentimento del poeta - quale fosse in quel momento lo stato d'animo di Dante («e quale allora femmi, / salsi colei che la cagion mi porse»); e prima, nel medesimo canto, in riferimento a Dio, che solo legge nei cuori umani (vv. 37-39: «Ed ella: "Se tacessi o se negassi / ciò che confessi, non fora men nota / la colpa tua: da tal giudice sassi!["]»).

Se così intendiamo, ammettendo cioè - come le parole stesse della donna suggeriscono - che il reale esecutore o mandante dell'assassinio, consumatosi nella remota Maremma, fosse rimasto ignoto, il caso della Pia è opposto, sotto questo riguardo, a quello di Omberto Aldobrandeschi ${ }^{20}$, che analogamente allude, senza descriverle, alle circostanze della sua morte («ogn'uomo ebbi in despetto tanto avante, / ch'io ne mori', come i Sanesi sanno, / e sallo in Campagnatico ogne fante» [Purg. XI, 64-66]), ma solo perché realmente a tutti note - almeno a Siena e a Campagnatico - e quindi non bisognose di indugio; e che, soprattutto, presenta questa morte come il degno, inevitabile e meritato esito della propria superbia nobiliare (vv. 67-68: «e non pur a me danno / superbia fa») $)^{21}$.

In tal modo, ne esce almeno in parte ridimensionato anche l'incanto musicale tradizionalmente attribuito ai quattro versi finali pronunciati dalla Pia, che - visti e letti in una diversa prospettiva esegetica e retorica - si rivelano improntati invece, nella loro strenua elaborazione formale (vicina, nella sua intarsiatura, a quella di certe concettose iscrizioni sepolcrali latine medievalii) ${ }^{22}$, non tanto ad una scorrevole fluidità melodica, quanto piuttosto ad una puntuta asciuttezza capace di racchiudere l'intera parabola esistenziale in un verso dalla nuda, fredda struttura speculare, e di muovere un'accusa precisa al marito, additandolo come l'impunito colpevole della sua uccisione e sottolineando con forza il venir meno da parte di lui all'impegno solennemente sancito col matrimonio ${ }^{23}$. Né si tratta di interpretazione infondata: è noto infatti che le anime dell'antipurgatorio - e non solo quelle dei morti per forza - si rivelano ancora in qualche misura legate agli affetti terreni, come dimostra, e non solo - di nuovo - da parte degli spiriti del quinto canto, la forte compassione di sé, manifesta nell'insistenza sull'oltraggio inflitto al loro corpo ${ }^{24}$ (né si cade nell'impressionismo, a mio avviso, se si sottolinea quanto questo sentimento di tragica compassione traluca in un verbo marcatamente 'espressivo' come il disfecemi impiegato dalla Pia per accennare alla propria morte) $)^{25}$.

\section{Filologia e biografia}

Alcuni ritengono ingiustificato (se non banalizzante) il parallelismo tra Pia e Francesca; eppure Dante stesso lo autorizza, e la certo non causale corrispondenza 'verticale' col quinto dell'Inferno (oltre al fatto che Pia è la prima donna dopo la Polentana a prendere la parola nel poema) è un indizio inequivocabile in tal senso, in un canto che esibisce un altro e ancor più stretto legame con un analogo episodio infernale ${ }^{26}$. Entrambe, anche se per ragioni diverse, sono reticenti; entrambe, anche se per ragioni diverse, alludono al marito obliquamente, senza nominarlo. Se Gianciotto è semplicemente l'uccisore di Paolo e di Francesca, e di lui si dice solo che la Caina lo attende, del marito la Pia si limita ad affermare che egli è l'unico a conoscere il modo della sua morte. La premurosa e 'femminile' sollecitudine dimostrata dalla Pia verso Dante (vv. 130-131: «Deh, quando tu sarai tornato al mondo / e riposato de la lunga via»), che non è poeticamente meno significativa perché riconducibile a moduli epigrafici ${ }^{27}$, suona come una captatio 
benevolentiae analoga a quella con cui Francesca si rivolge a Dante, prima definendolo «animal grazïoso e benigno» (Inf. V, 88), e poi addirittura evocando l'impossibile di una sua preghiera per il poeta («se fosse amico il re de l'universo, / noi pregheremmo lui de la tua pace» $[\mathrm{V}, 91-92])$. Captatio benevolentiae che, nel caso della Pia, è finalizzata a ottenere da Dante, una volta tornato sulla terra, le preghiere che abbrevierebbero il suo soggiorno antipurgatoriale (come, ad esempio, accadde a Forese Donati grazie alle preghiere della moglie Nella) ${ }^{28}$.

16 L'invocazione della donna («Ricorditi di me ${ }^{29}$ ) va spogliata, nuovamente, di buona parte del patetismo depositato su di essa da generazioni di lettori. Pia qui non chiede a Dante di essere ricordata ai suoi parenti, evidentemente perché la colpa da lei commessa la fa da loro ritenere irrimediabilmente dannata, a prescindere dal suo assassinio e dal suo pentimento (l'uno e l'altro rimasti ignoti agli uomini); cosicché solo Dante, che l'ha vista tra i salvati, potrà pregare Dio per lei. Infatti, a differenza di altre anime del Purgatorio, Pia non invita il poeta a «ridire» il «vero» ai «vivi» (come ad esempio fanno Manfredi, Bonconte e Sapia), perché i vivi potrebbero non credere a Dante, e in ogni caso non vorrebbero pregare per una peccatrice come lei. Tutto fa pensare, insomma, che la condotta disonesta della donna - di qualunque genere essa sia stata - abbia gettato discredito su di lei e sulla sua famiglia, e che per questo ella non chieda al poeta di adoperarsi per la sua postuma 'riabilitazione'; d'altra parte, Pia, ormai «pacificata» $(\mathrm{V}, 56)$, tace la casata e sua e anche quella del marito, per non gettare infamia su nessuna delle due.

Oltre questo - che, ripeto, è autorizzato dal testo - è arduo spingersi. Per farlo, dovremmo avere notizie sicure sul personaggio e sulla sua vita; e, come si sa, nessuna delle molte ricostruzioni proposte da storici, commentatori ed esegeti fin dal XIV secolo risulta davvero coerente e immune da dubbi e incertezze. Pochi e contradditori i documenti; impossibile farli quadrare con le informazioni fornite da Dante senza dover ricorrere a una ridda di ipotesi, spesso troppo onerose o scarsamente verosimili, tanto che, se non emergono nuovi dati, la certa identificazione storica della Pia e del suo sposo risulta di fatto impossibile. E senza notizie sicure, pressoché impossibile è anche sciogliere il nodo testuale e interpretativo degli ultimi due versi («salsi colui che 'nanellata pria / disposando [vel disposata] m'avea con la sua gemma»): è questo un caso in cui la filologia annaspa, perché solo la biografia potrebbe indicarle la strada giusta. La preferenza dei moderni editori per disposando si fonda infatti su due elementi tutt'altro che solidi: la convinzione che qui Dante alluda ai due momenti simultanei della cerimonia matrimoniale (l'atto di consentire in una donna come propria legittima moglie e l'anulatio $)^{30} \mathrm{e}$ il pregiudizio estetico secondo il quale l'allusione a un precedente matrimonio della Pia ('nanellata pria) costituirebbe una 'stonatura poetica' e fornirebbe un dato di cronaca che - anche qualora fosse documentabile o plausibile - risulterebbe inessenziale e superfluo ${ }^{31}$.

18 La macchinosa ridondanza (tanto più inspiegabile in un contesto caratterizzato dalla massima sintesi) di una tale descrizione del matrimonio è stata da più parti sottolineata ${ }^{32}$; e spiegarla con la volontà della donna di soffermarsi nostalgicamente sul momento felice delle sue nozze fa ricadere nell'impressionismo che tanto spesso ha guastato l'esegesi di questi versi. Inoltre, resta valida l'osservazione dello Scolari in merito alla sintassi decisamente infelice, contorta e anomala prodotta dalla lezione disposando (con l'ausiliare inserito a forza all'interno della gerundiva, come mai si verifica altrove in Dante) $)^{33}$. Ciò farebbe propendere per disposata, che può invocare 
anche il conforto dello stemma (essendo attestata da alcuni dei più autorevoli codici della famiglia toscana occidentale $b$ e di quella settentrionale $e)^{34}$, benché Giorgio Petrocchi ipotizzi una trafila disposando (disposādo) > disposato > disposata che renderebbe ragione del gerundio come lectio prior ${ }^{35}$. Ma il punto, si ripete, è che la prospettiva dalla quale guardare al problema deve essere capovolta: soltanto aver piena e sicura contezza della biografia della donna potrebbe sciogliere il dilemma della lezione, che i soli criteri interni del metodo non bastano a chiarire.

Accogliendo disposata ${ }^{36}$, in 'nannelata pria potremmo leggere l'allusione a un precedente matrimonio della Pia, la quale sarebbe stata dunque già stata sposata prima di contrarre nuove nozze con l'uomo che si sarebbe reso colpevole della sua morte in Maremma (uccidendola, o facendola uccidere, o recludendola e lasciandola morire d'inedia o di malaria); e poiché nessuna delle identificazioni proposte nel tempo con figure storicamente documentabili soddisfa, è incongruo affermare che questa ipotesi è contraddetta dalla storia ${ }^{37}$. Il dettaglio ci consegnerebbe il ritratto di una donna esperta del mondo, vedova, non più giovanissima, che come tale poteva essere maggiormente incline agli allettamenti dell'amore carnale e alla tentazione dell'adulterio; ma le controindicazioni a una simile lettura non mancano. In un contesto caratterizzato da estrema sintesi e concentrazione espressiva, l'aggiunta di una tale precisazione autobiografica da parte della Pia appare poco giustificata; inoltre, e soprattutto, il nesso tra la vedovanza, il successivo tradimento e la morte violenta della donna non è immediato né tanto meno automatico, e a stringerlo indebitamente potremmo essere indotti dall'impulso a spingere troppo sul pedale del parallelismo tra Pia e Francesca (come fa chi definisce senz'altro Pia «la Francesca del Purgatorio» ${ }^{38}$ ), evocando in maniera altrettanto forzata - complice il riferimento alla Maremma, ossia al castello e alle terre presumibilmente possedute dal marito in quella regione - scenari da amor cortese, con un pensiero parimenti indebito, magari, alla tragica storia di Guglielmo Rossiglione narrata da Boccaccio in una celebre novella del Decameron (IV, 9) ${ }^{39}$.

In un'altra e forse più fruttuosa direzione potrebbe condurci la proposta avanzata da Gino Casagrande ${ }^{40}$, che - optando a sua volta per la lezione disposata, giustamente intesa, secondo quanto dicevamo poc'anzi, come 'sposata', 'presa per moglie' - ritiene che 'nanellata pria si riferisca alla promessa di matrimonio (la locuzione sarebbe infatti «una condensazione semantica per indicare la cerimonia della desponsatio, al centro della quale è l'anello della promessa di nozze») e che il v. 136 alluda alla cerimonia nuziale vera e propria (che poteva essere celebrata anche uno o due anni più tardi) ${ }^{41}$. Casagrande suppone che la Pia si riferisca ai due momenti del suo matrimonio, ma - recuperando un'ipotesi di André Pézard - non esclude che con 'nanellata pria la donna alluda al fatto di essere stata in precedenza promessa in sposa ad un altro, e che tale promessa venisse poi annullata per consentire alla Pia di contrarre un diverso matrimonio ${ }^{42}$ : e, poiché il diritto canonico considerava peccato mortale la rottura della promessa matrimoniale (prevedendo specifica penitenza per chi se ne fosse macchiato), se responsabile della rottura fosse stata la donna, ciò, essendosi ella pentita solo al momento della morte, potrebbe essere la causa della sua presenza nell'antipurgatorio ${ }^{43}$. La precisazione 'nanellata pria, in tal caso, non sarebbe superflua, giacché da un lato renderebbe ragione della colpa della Pia (pur senza esplicitarla, come avviene anche per gli altri personaggi di questi canti), e dall'altro accrescerebbe l'odiosità del crimine del marito, il quale (per ragioni non specificate e sulle quali vano sarebbe speculare: smodata gelosia? desiderio di risposarsi con un'altra donna?) avrebbe ucciso o fatto 
morire la moglie, benché ella avesse infranto per amore di lui una precedente promessa di matrimonio.

21 In ogni caso, con la lezione disposata il dettaglio fornito al v. 135 ('nanellata pria) viene ad acquistare una sua precisa funzione; e l'autoritratto della Pia ne esce decisamente più mosso e complesso, giacché quella lezione, facendo entrare in gioco - sia pure oscuramente - il passato della donna ${ }^{44}$, arricchisce il suo profilo esistenziale e morale, ulteriormente ripulendolo dalle scorie sentimentalistiche e patetiche accumulatevi dalla tradizionale esegesi ${ }^{45}$, e rendendo ragione della sua presenza nell'antipurgatorio, oltre che del riserbo con cui ella si rivolge a Dante e (tacendo dei propri familiari) richiede le sue preghiere.

\section{Pia e le altre}

Il nome della Pia è contenuto in quelli di Sapia e Piccarda, a suggello di caratteri e destini in parte comuni. Senese anch'essa, Sapia sembra da un lato differenziarsi dalla sua concittadina dell'antipurgatorio per la loquacità (il dialogo tra lei e Dante occupa ben 42 versi) e per una passionalità che ancora ritiene qualcosa dell'asprezza originaria, come ben emerge dalla rievocazione della battaglia di Colle (conclusa riferendo il suo gesto blasfemo di sfida a Dio, che ricorda quelli di Capaneo e di Vanni Fucci, oltre che - tra i personaggi della mitologia e della letteratura - di Niobe) e dalla conclusiva maledizione profetica ai danni dei Senesi. E se davvero pietosa - verso il poeta e, in parte, verso il marito - è la $\mathrm{Pia}^{46}$, fin da subito Sapia sottolinea invece la natura intimamente antifrastica del suo nome, che avrebbe dovuto importare saggezza (vv. 109-110: «Savia non fui, avvegna che Sapìa / fossi chiamata»). Nondimeno, al di fuori dell'influenza esercitata sul lettore dall'esegesi tradizionale che insiste oltre misura sulla 'delicatezza' dei versi a lei attribuiti, anche la Pia fu peccatrice fino all'ultim'ora, e niente vieta di pensare che anche i suoi peccati fossero, se non «orribili», quantomeno "gravi»; così come, d'altro canto, il pentimento e la pacificazione addolciscono l'animo di Sapia, che quando apprende Dante essere vivo, si congratula della grazia divina a lui concessa (v. 146: «gran segno è che Dio t'ami»), lo prega di ricordarla ai vivi «per quel che tu più brami» (v. 148) ed evoca nostalgicamente la comune patria (v. 149: «se mai calchi la terra di Toscana»). E se Pia non ha nessuno al mondo da cui possa sperare preghiere, Sapia confida di poter essere "rinfamata" - in senso spirituale, s'intende - presso i suoi «propinqui» (v. 150).

Se è poi eccessivo parlare di Piccarda come di una sorta di Pia del paradiso, le affinità tra i due personaggi e tra le loro vicende terrene sono però evidenti. Anche Piccarda è stata vittima di una violenza maschile (quella del fratello, che a forza la cavò, per obbligarla a contrarre un matrimonio 'politico', dal monastero di Santa Chiara a Firenze); anch'ella non fa il nome del parente che le fece oltraggio e che determinò il suo destino ultraterreno (parlando anzi, genericamente, solo di «uomini [...] a mal più che a bene usi» [Par. III, 106]); anch'ella vela di reticenza i dettagli della sua storia («Iddio si sa qual poi mia vita fusi» [III, 108]), con una formula analoga a quella adottata dalla Pia nei confronti del marito e del delitto da lui commesso ${ }^{47}$. E anche Piccarda esaurisce la sintesi della sua esperienza terrena in sei versi, bipartiti - come nel caso della Pia - tra la rievocazione dei tempi felici (per lei, quelli della scelta monastica e dei voti: un altro genere di matrimonio dunque, quello con Dio, per il quale ella fuggì dal mondo, per vegliare e dormire «con quello sposo ch'ogne voto accetta» [III, 101]) e la 
sobria rievocazione dell'oltraggio che intervenne a por fine, inaspettatamente, a quella effimera felicità («Uomini poi...» [III, 106-108]).

Ma un'altra, inattesa figura femminile si affaccia in controluce nell'epigrafe o autoepitaffio della Pia, che Dante confeziona nella forma di quattro endecasillabi a rima alterna, e che qui finalmente si trascrive nella lezione a mio avviso preferibile:

Ricorditi di me, che son la Pia:

Siena mi fé, disfecemi Maremma.

Salsi colui che, 'nanellata pria,

disposata m'avea con la sua gemma.

Versi impreziositi da un elaboratissimo gioco di rime interne (che: fé: che; 'nanellata: disposata ${ }^{48}$, allitterazioni (son - Siena - Salsi, disfecemi - disposata), consonanze (pria avea - sua), paronomasie in rima (Pia: pria) e, soprattutto, emistichi a specchio con figura etimologica a stretto contatto ${ }^{49}$ (Siena mi fé/ disfecemi Maremma), insistito mitacismo e duplice, fortissima antitesi: fortissima non solo perché Siena e la Maremma, situate a mo' di antipodi alle estremità del verso, si connotano per la donna l'uno come luogo di memorie felici (la giovinezza, il matrimonio) e l'altro come luogo di dolore e di morte, ma anche perché i loro nomi evocano inevitabilmente idee accessorie che chiamano in causa l'opposizione tra civiltà e barbarie (in virtù della trista fama che accompagnava la Maremma per la sua natura di terra aspra e selvaggia, oltre che insalubre $)^{50}$, associandosi rispettivamente - se nel dir questo non si è condizionati oltre misura dalle raffigurazioni romantiche della Pia che campeggiano in ritratti ottocenteschi come quelli di Eliseo Sala, Stefano Ussi e Dante Gabriel Rossettia immagini di elegante vita cittadina e di lugubre, malinconica solitudine ${ }^{51}$.

Ebbene, in questo capolavoro di retorica sepolcrale spicca, nella posizione apicale della chiusa, il nome della moglie di Dante. Della sua vita e dei suoi spostamenti negli anni dell'esilio del marito niente sappiamo; benché da più parti, e da tempo, si ritenga infondata la convinzione di Boccaccio che ella non abbia mai seguito Dante e i figli (e si respinga, come dettata da convenzionale misoginia, l'immagine negativa di lei e dei suoi rapporti col poeta offerta dal Certaldese $)^{52}$, restano solo supposizioni quelle di quanti inclinano a pensare che - attestata essendo la sua presenza a Firenze solo dopo la morte del poeta - Gemma si sia riunita alla famiglia almeno in alcune fasi e in alcuni luoghi dell'esilio, a Lucca, a Bologna o a Ravenna ${ }^{53}$. Certo, invece, è che Dante mai la nomina nei suoi scritti; è possibile, come di recente si è supposto, che a lei il poeta alluda celatamente, e affettuosamente, in due luoghi della Commedia ${ }^{54}$, mentre è da considerare inaccettabile l'ipotesi (avanzata con cautela da Claudio Giunta e poi accolta senza riserve da Marco Santagata $)^{55}$ che a Gemma si faccia riferimento nella quinta stanza della canzone Tre donne, dato che - a norma della tradizione lirica medievale lessico e tono di quegli appassionati versi non sembrano potersi usare per la propria consorte, cui, di conseguenza, a nessun lettore coevo sarebbe certo venuto in mente di ricondurli ${ }^{56}$.

Qui, però, il contesto, ossia l'evocazione della figura della Pia, sembra autorizzare a non considerare del tutto arbitraria l'ipotesi di un fugace, discretissimo cenno del poeta a Gemma Donati ${ }^{57}$. Un cenno 'pietoso', un saluto nascosto, un gesto risarcitorio da parte di un marito che, come quello della Pia, tanto dolorosamente ha inciso sul destino della propria sposa: quella Gemma che a sua volta, come la misteriosa donna senese, spesso sarà stata indotta a confrontare il felice tempo della giovinezza e le liete speranze da lei riposte nel matrimonio con la ben diversa realtà degli anni successivi e dell'età matura, 
segnata senza rimedio da distacco, lontananza e solitudine. In questa luce, il dolce pensiero rivolto dalla Pia a Dante ai vv.130-131 («Deh, quando tu sarai tornato al mondo / e riposato de la lunga via») acquista una sfumatura più intima e precisa: pensiero di sollecitudine non tanto materna, come talora si è detto ${ }^{58}$, quanto piuttosto maritale, di moglie in ambasce per la «lunga via» del proprio sposo esule, cui chiede, in memoria della ormai lontana promessa matrimoniale, almeno la fedeltà perenne del ricordo.

\section{NOTE}

1. Che a partire dal Lana lo identificano con tale "Giampolo» o «Zanpòlo», toscanamente «Ciàmpolo» (cfr. Iacomo della Lana, Commento alla 'Commedia', a cura di M. Volpi, con la collaborazione di A. Terzi, vol. I, Roma, Salerno Editrice, 2009, pp. 638-639).

2. Benvenuti de Rambaldis Comentum super Dantis Aldigherii Comoedia, curante J. Ph. Lacaita, vol. II, Florentiae, Typis G. Barbèra, 1887, p. 135.

3. Osservazione che vale, in realtà, per molte delle anime del Purgatorio, anche al di fuori dell'antipurgatorio. Per non parlare di fenomeni ancora più eclatanti come la conversione cristiana di Stazio, probabilmente dedotta - al pari della lussuria di Guinizzelli e Arnaut Daniel, ad esempio - dalle sue opere. Irricevibile l'ipotesi avanzata da A. Soro, Un'interpretazione del personaggio di Pia di 'Purg.' V 130-136, «Electronic Bulletin of the Dante Society of America», 2009, pp. non numerate, consultabile all'indirizzo internet <http://www.princeton.edu/ dante/ebdsa/ soro042109.html>, che la Pia sia allegoria e personificazione della pietas.

4. G. Inglese, commento a D. Alighieri, Purgatorio, revisione del testo e commento di G. I., Roma, Carocci, 2011, p. 88.

5. Vd., proprio a proposito della Pia, quanto scriveva M. Barbi, La Pia di Dante ('Purg.', V 130-136) [1937], in Id., Con Dante e coi suoi interpreti, Firenze, Le Monnier, 1941, pp. 336-341, a p. 336: «[...] a intendere la poesia di Dante non occorre più di quel tanto ch'egli ci ha detto».

6. Cfr. al riguardo M. Tavoni, Qualche idea su Dante, Bologna, Il Mulino, 2015, pp. 209-210, che sottolinea l'«altissimo tasso di implicitezza» proprio della Commedia, e osserva: «Spesso, ma non sempre, l'identità dei personaggi e il senso delle loro parole diventa chiaro nel séguito dell'incontro, ma mai e poi mai Dante autore si sente in dovere di fornirci tutta l'informazione necessaria per capire per filo e per segno che cosa è accaduto e, in definitiva, tutta l'informazione necessaria per capire ciò che lui ci ha voluto dire raccontandoci - o meglio, con le sue parole, ridicendoci- ciò che ha visto» (corsivi suoi). Lo stesso Tavoni (ivi, p. 210) porta l'esempio dell'anonimo suicida fiorentino che compare alla fine del XIII dell'Inferno, contestando l'opinione di quanti ritengono che il personaggio non sia identificabile e che il poeta stesso non richieda al lettore la sua identificazione.

7. Cfr. al riguardo le celebri pagine di E. Auerbach, Dante, poeta del mondo terreno [1929], in Id., Studi su Dante, prefazione di Dante Della Terza, Milano, Feltrinelli, 1999, pp. 3-161, alle pp. 129-136 (dove è recato, alle pp.132-133, anche l'esempio della Pia, riguardo alla quale si dice che «non ci manca nulla: essa è perfettamente reale e evidente»); e sulla sua scorta C. Giunta, Lingua e generi della 'Commedia', in E. Menetti e C. Varotti (a cura di), La letteratura e la storia, Bologna, Gedit, 2007, pp. 149-167, a p. 157. 
8. E. Auerbach, Dante, poeta del mondo terreno, cit., p. 132, dove si aggiunge che tale «cifra» riceve la sua ricchezza «dalla massa di avvenimenti che comprende, e da cui è ricavata; gli avvenimenti sono espressi soltanto in minima parte; ma ciò che è espresso è l'elemento decisivo, e quanto è tralasciatovi è contenuto e risuona in esso».

9. All'analisi delle numerose e complesse questioni strutturali, teologiche e morali poste da questa sezione del poema è in buona parte dedicato il volume di G. Sasso, Purgatorio $e$ antipurgatorio. Un'indagine dantesca, Roma, Viella, 2019 (in particolare il cap. Purgatorio $e$ antipurgatorio: questioni di struttura, pp. 11-152). Ancora utile la voce Antipurgatorio dell'Enciclopedia dantesca, vol. I, Roma, Istituto della Enciclopedia Italiana, pp. 304-306 (curata da S. Pasquazi), disponibile anche in rete (<www.treccani.it/enciclopedia/antipurgatorio>).

10. Senza arrivare a parlare di una rigida suddivisione dell'antipurgatorio in quattro 'gironi' (secondo la proposta non condivisibile di G. Poletto nel suo commento al Purgatorio, Roma / Tournay, Tip. liturgica di San Giovanni / Desclée, Lefebvre \& $C^{\text {ie }}, 1894$, p. 60), pare indubbio che in questa zona del secondo regno le anime siano raggruppate in quattro distinte schiere. G. Sasso, Purgatorio e antipurgatorio. Un'indagine dantesca, cit., pp. 23-26 e 29, ritiene che solo due schiere siano precisamente caratterizzate (quelle degli scomunicati e dei morti di morte violenta), mentre pigri e 'negligenti' - nel senso stretto del termine - non costituirebbero gruppi altrettanto definiti (eccezion fatta per i principi della valletta). Ma i pigri sono descritti come una vera e propria schiera («ivi eran persone / che si stavano a l'ombra dietro al sasso» [IV, 103-104]), e tra i 'negligenti' il solo Sordello (insieme, forse, agli altri negligenti «per eccesso di opere», come li definisce Inglese nel suo commento al Purgatorio, cit., p. 105) sembra stare in disparte e avere libertà di movimento.

11. Ancora Sasso (Purgatorio e antipurgatorio. Un'indagine dantesca, cit., p. 27) non vede un'anticlimax di gravità nelle quattro tipologie di negligenza punite nell'antipurgatorio; eppure la superiore colpevolezza degli scomunicati pare evidente (e, ripeto, è simboleggiata dalla loro stessa collocazione), così come quella dei pigri rispetto a quella dei morti per forza (che la violenza subita obbligò al pentimento in extremis). D'altronde, non si comprende la ragione per cui Dante avrebbe dovuto sottrarre l'antipurgatorio alla legge che regola l'intero secondo regno, in base alla quale la gravità dei peccati decresce man mano che si sale verso la vetta; né può dubitarsi, sulla base delle indicazioni fornite dal poeta, che le quattro schiere vengano incontrate dai due pellegrini nel corso della loro ascesa verso il purgatorio vero e proprio.

12. Summa theol., $\mathrm{II}^{\mathrm{a}}-\mathrm{II}^{\mathrm{ae}}$, q. 54, a. 2.

13. Così G. Sasso, Purgatorio e antipurgatorio. Un'indagine dantesca, cit., p. 41.

14. Purg. III, 78.

15. Vd. ora al riguardo V. L. Puccetti, Fuga in 'Paradiso'. Storia intertestuale di Cunizza da Romano, Ravenna, Longo, 2010, soprattutto pp. 9-53. La golosità di Belacqua si deduce per antifrasi dal suo nomignolo (Be[vi]lacqua).

16. Davvero fuori luogo, pertanto, parlare ad es. di questi versi come una sorta di «anticipazione del Paradiso» (così G. Cambon, Le modulazioni della sollecitudine nel V del 'Purgatorio', «Ausonia», vol. XXV, 1970, pp. 9-18, a p. 9).

17. «pentendo e perdonando, fora / di vita uscimmo a Dio pacificati» (Purg. V, 55-56).

18. A. Chiari, La Pia, in Id., Ancora con Dante, Napoli, Liguori, 1977, pp. 135-141, alle pp. 137-138, scrive che quei versi «racchiudono un'accusa» che è "piuttosto un rimprovero per il marito»; e così anche Daniele Mattalia (D. Alighieri, La Divina Commedia, commento di D. M., II [Purgatorio], Milano, Rizzoli, 1975, p. 113): «[...] rancore non c'è, indubbiamente, e la disposizione della Pia pare al perdono; ma, pur in modo velato, la messa a punto della responsabilità è, dicevamo, precisa [...]: una formale accusa, né più né meno». Che il marito, nella primavera del 1300, fosse ancora vivo è implicito nelle parole della Pia, come osserva già P. Armour, Words and the Drama of Death in 'Purgatorio' V (1993), in J. C. Barnes e J. Petrie (eds), Word and Drama in Dante. Essays on the 'Divina Commedia', Dublin, Irish Academic Press, 1993, pp. 93-122, a p. 112. 
19. Così non pochi tra i commentatori antichi, come ad es. il Lana, l'Ottimo, l'anonimo autore delle cosiddette Chiose cassinesi («qui [scil. Nello Pannocchieschi] eam occidit ita secrete quod numquam aliquis scivit et ideo dicit quod ille scit qui eam disponsavit et non alius»: Il codice cassinese della 'Divina commedia', per la prima volta letteralmente messo a stampa per cura dei monaci benedettini della Badia di Monte Cassino, Monte Cassino, Tipografia della Badia di Monte Cassino, 1865, p. 215) e Francesco da Buti. Giovanni Andrea Scartazzini (nel suo commento alla Commedia, vol. II [Purgatorio], Leipzig, Brockhaus, 1875, p. 62) osserva: «Non si vorrà negare che le parole Salsi colui parlano in favore di questi espositori». Per un panorama delle glosse dei primi commentatori a questo episodio cfr. G. Varanini, Il punto sulla Pia ('Purg.', V, 130-136), in Studi filologici letterari e storici in memoria di Guido Favati, Padova, Antenore, 1977 (poi in Id., L'acceso strale. Saggi e ricerche sulla 'Commedia', Napoli, Federico \& Ardia, 1984, pp. 108-122), pp. 621-638, alle pp. 621-627.

20. Cui invece lo avvicina M. Porena, commento a D. Alighieri, Purgatorio, nuova ed. riveduta e ampliata, Bologna, Zanichelli, 1967, p. 107, seguito ad es. da G. Inglese, commento al Purgatorio, cit., p. 150.

21. Contro fa di Triv e Urb (accolto da Petrocchi: vd. la sua difesa della lezione in D. Alighieri, La Commedia secondo l'antica vulgata, seconda ristampa riveduta, vol. III, Firenze, Le Lettere, 1994, p. 181), Inglese adotta qui fu (di Eg Fi Parm Po Pr), che pare in effetti poziore; altri (fra cui Casella, Vandelli e Porena) optarono per fé, che è lezione ignota ai codici dell'antica vulgata.

22. Come ad es., per rimanere al quinto canto purgatoriale, quella di Iacopo del Cassero incisa sul suo sepolcro nella chiesa di San Domenico a Fano, riportata dallo Scartazzini nell'editio maior del suo commento al poema (vol. II, cit., p. 55) e forse nota a Dante (vd. G. Fallani, Dante e la cultura figurativa medievale, Bergamo, Minerva Italica, $1976^{2}$, pp. 185-188; M. Picone, Canto V, in Lectura Dantis Turicensis. Purgatorio, Firenze, Cesati, 2001, pp. 71-83, alle pp. 81-82).

23. Anche C. Giunta, Lingua e generi della 'Commedia', cit., p. 155, sottolinea la «fredda oggettività dei tre versi conclusivi». L'osservazione di A. Jacomuzzi, Il canto V del 'Purgatorio' [1976], in Id., L'imago al cerchio e altri studi sulla 'Divina Commedia', Milano, FrancoAngeli, 1995, pp. 215-230, a p. 230, che parla di «stile tragico» riguardo ai due versi finali (sottolineando la loro «terribile gravità»), deve essere estesa all'intero autoepitaffio della Pia, che occupa gli ultimi quattro versi del canto (vd. infra, § 4).

24. Compassione, e insistenza, che sono due aspetti di quello che Picone, riferendosi alle anime dell'antipurgatorio, ha definito «complesso di Palinuro» (vd. M. Picone, Canto V, cit., pp. 77-80).

25. Vd. oltre, $\$ 4$ e nota 49. Il TLIO-Tesoro della Lingua Italiana delle Origini (all'indirizzo internet $<$ http://tlio.ovi.cnr.it/>), s.v., elenca, prima di quelli danteschi, solo tre esempi di disfare nel senso di 'far morire', 'uccidere', nessuno dei quali toscano (l'Elegia giudeo-italiana, l'Istoria dello Pseudo-Uguccione, i Sermoni subalpini, rispettivamente di area mediana, lombarda e francopiemontese). Come scrive L. Fiorentini, Lettura del V canto del 'Purgatorio' di Dante, in L. Cesalli et al. (a cura di), Ad placitum. Pour Irène Rosier-Catach, vol. I, Ariccia, Aracne, 2021, pp. 287-297, a p. 297, nella Pia «il dolore per quell'oltraggio terribile non è ancora venuto meno: è un'ombra che ancora la tormenta, riportandola di necessità a quel momento doloroso e decisivo per la sua esistenza terrena e ultraterrena».

26. Mi riferisco ovviamente alle vicende parallele e speculari - e dunque opposte quanto all'esito ultimo - di Guido da Montefeltro (Inf. XXVII) e di suo figlio Bonconte (Purg. V).

27. Cfr. G. Gorni, Epitaffi nella 'Commedia', «Studi danteschi», vol. LXVIII, 2003, pp. 1-16, a p. 12; C. Giunta, Lingua e generi della 'Commedia', cit., pp. 154-158; S. Carrai, Quando ti gioverà dicere «I' fui». Lo stile epigrafico nella 'Commedia' [2010], in Id., Dante e l'antico. L'emulazione dei classici nella 'Commedia', Firenze, Sismel-Edizioni del Galluzzo, 2012, pp. 43-86, alle pp. 52-54.

28. Purg. XXIII, 88-90.

29. L'uso impersonale, al congiuntivo presente, di ricordare (frequente nella lingua antica e anche altrove attestato nel poema, ad es. in Inf. IX, 98) vale ad attenuare la forza dell'esortazione. 
30. Sulla questione vd. da ultimo G. Casagrande, 'Purgatorio' V: «inanellare» / «disposare», "Studi danteschi», vol. LXII, 2007, pp.45-63, alle pp. 48-56, che mostra come, con la lezione disposando (che vale 'nell'atto di farmi sua sposa', come già glossava M. Barbi, Per una più precisa interpretazione della 'Divina Commedia', in Id., Problemi di critica dantesca. Prima serie (1893-1918), Firenze, Sansoni, 1934, pp. 197-303, a p. 279), i due momenti costituiscano i cosiddetti sponsalia de praesenti, ossia la cerimonia matrimoniale propriamente detta. Impropria la rubricazione di questo verso dantesco tra gli esempi di disposare nel senso di «legarsi con solenne promessa prematrimoniale, fidanzarsi (tramite la cerimonia della desponsatio)» che si trova nel TLIO, dove pure è testimoniata ed esemplificata l'accezione «prendere in matrimonio». Anche Bruno Basile (disposare, in Enciclopedia dantesca, cit., II, pp. 507-508) intende disposando come 'legandosi a me con solenne promessa' o 'dichiarando di volermi per moglie'.

31. Ma, come osserva giustamente G. Varanini, Il punto sulla Pia, cit., pp. 635-636, «diremmo che, a ben vedere, l'intensità poetica dell'episodio non risulti offuscata [...] ove si accetti un'interpretazione diversa da quella tradizionale. D'altronde, optare per una proposta esegetica solo perché questa sembri meglio preservare la poesia può risultare criticamente scorretto».

32. Cfr. ad es. ivi, p. 631, e P. Jodogne, Dante, 'Purgatorio' V 136: per la lezione "disposata», in A. Paolelli (a cura di), Miscellanea di studi danteschi in memoria di Silvio Pasquazi, vol. I, Napoli, Federico \& Ardia, 1993, pp. 441-446, a p. 445 (che osserva come il sintagma inanellare con una gemma risulti tautologico, giacché la gemma faceva parte dell'anello); Inglese, p. 89, parla di «distinzione [scil. tra i due momenti descritti ai vv. 135 e 136] poco motivata, se non proprio viziosamente burocratica»; né mi pare che la ridondanza scompaia se si suppone trattarsi di un medesimo atto (parafrasando: "mi aveva dato il suo anello prendendomi in moglie"), riferendo pria al tempo felice dell'amore e del matrimonio, in contrapposizione al «delitto consumatosi in Maremma» (così ancora Inglese, ibid.; e anche Saverio Bellomo nel suo commento al Purgatorio, Torino, Einaudi, 2019, p. 79).

33. Cfr. A. Scolari, Nota dantesca (Pg. V 134-136), «Atti dell'Istituto veneto di scienze, lettere ed arti», vol.CXXI, 1962-1963, pp.147-155, alle pp.153-154 («Quando, nella Commedia, sia nelle forme passive e sia nei tempi composti, il participio precede l'ausiliare, Dante, tra l'uno e l'altro, non inserisce mai una subordinata, né esplicita né implicita»), con cui concorda P. Jodogne, Dante, 'Purgatorio' V 136: per la lezione «disposata», cit., p. 445. Nessuna reale obiezione, se non di natura estetica, fu portata a queste osservazioni da Francesco Maggini nella sua recensione al saggio dello Scolari (pubblicata postuma sul «Giornale storico della letteratura italiana», vol. CXLI, 1964, pp. 125-126). Considerare difficilior, in quanto «insolita», la sintassi generata dalla lezione disposando (come fa ad es. G. Ciavorella, 'Purgatorio' V: Iacopo del Cassero, Bonconte da Montefeltro, Pia dei Tolomei, «Esperienze letterarie», vol.XXXVIII, 2013, pp. 43-72, a p. 69) significa servirsi impropriamente di quel criterio ecdotico - come, purtroppo, non di rado si fa - per avallare qualunque lezione bizzarra e priva di attestazioni.

34. Mi riferisco allo stemma di Petrocchi; si tratta dei mss. Ash, Ham e (su rasura) Fi (della famiglia $b$ ) e $\mathrm{Rb}$ Urb (della famiglia $e$ ), cui si aggiungono Co Laur Mad Po (che leggono disposato, sintatticamente e semanticamente equivalente). In termini puramente aritmetici, le due lezioni sono rappresentate in misura pressoché equivalente nella tradizione antica (vd. G. Casagrande, 'Purgatorio' V: «inanellare» / «disposare», cit., p. 49; ne risulta, come scrive P. Jodogne, Dante, 'Purgatorio' V 136: per la lezione «disposata», cit., p. 443, che «la tradizione manoscritta non è categorica nell'assecondare la scelta tradizionale di disposando e riconosce come altrettanto legittima la lezione disposata»). A favore di disposata si pronunciò già lo Scartazzini (II, p. 63), con argomentazioni forse esposte in maniera un po' spiccia, ma a mio avviso più che ragionevoli; d'altronde, fra Sette e Ottocento disposata (o disposato) raccoglieva il favore di gran parte degli editori e degli esegeti, tra cui Dionisi, Bianchi, Fraticelli, Parenti, Viviani, Scarabelli, Fanfani, Campi (vd. in proposito l'ampia nota ad loc. di quest'ultimo: D. Alighieri, La Divina Commedia, 
ridotta a miglior lezione [...] per cura di G. Campi, vol. II [Purgatorio], Torino, Unione tipograficoeditrice, 1891, pp. 108-110).

35. D. Alighieri, La Commedia secondo l'antica vulgata, cit., vol. I (Introduzione), p. 194.

36. Come, tra i moderni editori, fa il solo Sanguineti (Dantis Alagherii Comedia, ed. critica per cura di F. Sanguineti, Firenze, Edizioni del Galluzzo, 2001, p. 215), che a norma bédieriana esempla il suo testo sulla testimonianza dell'Urbinate.

37. Le ricostruzioni dei retroscena politici della vicenda proposte da P. Armour, Words and the Drama of Death in 'Purgatorio', cit., pp. 113-115, e da M. Santagata, Le donne di Dante, Bologna, Il Mulino, 2021, pp.159-166, accumulano supposizioni non verificabili e talora, soprattutto nel secondo caso, decisamente 'fantapolitiche' e 'romanzesche'.

38. Così Mattalia nel suo cit. commento al poema, II, p. 111; ma gran parte dei commentatori moderni ritiene probabile o certo che la Pia fosse un'adultera assassinata per vendetta dal marito. Sulla questione vd. ora G. Masi, Dalla Bibbia, alla Pia, ad Amleto: ricordati dime, «Italianistica», vol. L, 2021, pp. 156-168, alle pp. 158-159. Nel suo album Pia come la vedo io (2007), Gianna Nannini immagina invece Pia come vittima innocente di una falsa accusa di adulterio cui il marito Nello, accecato dalla gelosia, volle prestare fede; tesi implicita nelle ricostruzioni di vari commentatori (e storici) antichi, a cominciare da Benvenuto da Imola.

39. Cfr. M. Barbi, La Pia di Dante ('Purg.', V 130-136), cit., p. 339: «Né il dire Dante che la Pia senese e i suoi compagni di pena furon "peccatori infino all'ultim'ora" giustifica la supposizione che s'alluda a questi supposti amori della Pia». Su questa linea, come si sa, il Bandello imbastì la sua nota novella di Pia de' Tolomei (Un senese truova la moglie in adulterio e la mena fuori e l'ammazza: parte I, nov. 12); per le sue numerose propaggini moderne e contemporanee vd. I. Gallinaro, "Ricorditi di me, che son la Pia», «Lettere italiane», vol. LXVII, $\mathrm{n}^{\circ} 3$, 2015, pp.469-499, alle pp. 470-472; E. D’Angelo, «Siena mi fé, disfecemi Maremma»: ritratti di Pia, da Dante a Cammarano, in G. Donizetti, Pia de' Tolomei, Venezia, Teatro La Fenice, 2005 («La Fenice prima dell'opera», 2004-2005, 7), pp. 23-45.

40. G. Casagrande, 'Purgatorio' V: «inanellare» / «disposare», cit., pp. 61-63.

41. Donde il doppio anello: quello semplice, consegnato alla donna dal promesso sposo, in qualità di pegno, negli sponsali con verba de futuro, e quello gemmato, messole al dito nel corso della cerimonia nuziale.

42. G. Casagrande, 'Purgatorio' V: «inanellare» / «disposare», cit., p. 57; e Dante, CEuvres complètes, traduction et commentaires par A. Pézard, Paris, Gallimard, $1983^{5}$, pp. 1150-1151 (dove tuttavia si ritiene più probabile che la promessa originaria fosse venuta meno a causa della morte dell'uomo). La vecchia ipotesi che 'nanellata pria alluda a una promessa di matrimonio fatta per procura, e che il procuratore (un famiglio del marito) sia stato poi l'esecutore materiale del delitto (così A. Lisini e G. B. Bandinelli, La Pia dantesca, Siena, Accademia per le Arti e per le Lettere, 1939, pp. 18-19, 53-55, 70-71, riprendendo e sviluppando a forza di supposizioni indimostrabili quanto sostiene l'anonimo autore delle glosse trasmesse dal Laur. XL, 7 - indicato per errore dagli studiosi come XV, 7 - a c. $87 \mathrm{v}$, dove al v. 136 ricorre la lezione disponsata) è stata ripresa di recente, in termini leggermente diversi e accogliendo disposata, da S. Pagani, «Ricorditi di me». Pia de' Malavolti e Nello de' Pannocchieschi ('Purg.' 5, 130-136), «Rivista di letteratura italiana», vol. XLIV, 2015, pp. 131-148, che identifica la donna con Pia de' Malavolti, sposa in prime nozze di Bertoldo (Tollo) degli Alberti e poi moglie di Nello Pannocchieschi, che, già procuratore di Tollo, dopo la morte di lui si sarebbe unito in matrimonio con la vedova.

43. Vd. ancora G. Casagrande, 'Purgatorio' V: «inanellare»/ «disposare», cit., pp. 58-59 (che aggiunge: «Non sarà stato mica questo il peccato della Pia?»).

44. P. Jodogne, Dante, 'Purgatorio' V 136: per la lezione «disposata», cit., p. 446, scrive che disposata crea in questi versi una «tensione che poeticamente s'allarga e drammatizza la vicenda della Pia, aprendo alla fantasia la possibilità d'intuire intime ragioni di contrasto tra questa donna (peccatrice) e colui che della sua morte violenta seppe la verità». 
45. Anche P. Armour, Words and the Drama of Death in 'Purgatorio', cit., p. 113, osserva che, a ben guardare, l'episodio della Pia «emerges as less sentimental than is generally believed and indeed acquires a more characteristical, darker, and even polemical tone».

46. Nome davvero 'parlante', il suo, come del resto quello di Bonconte, entrambi nomina omina; e con Bonconte la Pia ha pure in comune il fatto che anche di lei potrebbe dirsi «che non si seppe mai sua sepultura» $(\mathrm{V}, 93)$.

47. Fini osservazioni sul parallelismo Pia/ Piccarda ha soprattutto A. Chiari, La Pia, cit., pp. 140-141.

48. Anche in filologia, molto dipende dal punto di vista: a chi ritiene disposata una banalizzazione, scaturita da un originario disposando, e influenzata dal vicino 'nanellata (vd. ad es. E. Malato, «[...] per una lagrimetta che'l mi toglie». Lettura del canto V del 'Purgatorio', in Id., Nuovi studi su Dante. "Lecturae Dantis», note e chiose dantesche, Cittadella [PD], Bertoncello Artigrafiche, 2020, pp. 11-46, a p.43), si potrebbe appunto obiettare che disposata - a parte ogni altra considerazione di carattere sostanziale o sintattico, come quelle qui avanzate in precedenza - assicura la preziosa rima interna col verso precedente, che viene a perdersi adottando l'altra lezione, e che è ben congruente col ricco tessuto retorico dei quattro versi conclusivi del canto. Quanto all'altra rima interna, è vero che i due che non cadono in corrispondenza di accenti metrici, ma almeno il secondo (v. 135) acquista indubbio rilievo una volta considerato e letto 'nanellata pria come un inciso.

49. Esattamente come in Inf. VI, 42: «tu fosti, prima ch'io disfatto, fatto» (meno forte in senso 'fisico' è il senso di disfare in Par. XVI, 76: «udir come le schiatte si disfanno»).

50. Sottolineata anche da Dante, rispettivamente, in Inf. XIII, 7-9 e XXV, 19-21, e in Inf. XXIX, 48.

51. Non si tratta dunque solo di una «asciutta arguzia opposizionale» (come scrive E. Sanguineti, Dante reazionario, Roma, Editori Riuniti, 1992, p. 167). Proprio la rigorosa struttura chiastica del verso e la figura etimologica in forte rilievo attenuano la dipendenza dal pur indubbio precedente costituito dall'epitaffio di Virgilio riportato da Elio Donato («Mantua me genuit, Calabri rapuere», dove comunque il chiasmo è prodotto da quanto segue: «tenet nunc / Parthenope»: cfr. G. Parenti, Schemi classici nel V canto del 'Purgatorio', «Studi danteschi», vol. LIV, 1982, pp. 71-98, a p.98), anche perché siamo in presenza di un diffuso modulo epigrafico, che «oppone [...] la nascita in un dato luogo e la morte in un luogo straniero» (c. Giunta, Lingua e generi della 'Commedia', cit., p. 155).

52. Cfr. G. Boccaccio, Trattatello in laude di Dante, a cura di M. Fiorilla, in Le vite di Dante dal XIV al XVI secolo. Iconografia dantesca, a cura di M. Berté et al., Roma, Salerno Editrice, 2017, pp. 28-154, alle pp. 50 (I red.) e 128-129 (II red.), con le relative note del curatore.

53. Vd. al riguardo le considerazioni di M. Barbi, Un altro figlio di Dante (1922), in Id., Problemi di critica dantesca. Seconda serie (1920-1937), Firenze, Sansoni, 1941, pp.347-370, a p.357; e di M. Santagata, Le donne di Dante, cit., pp. 32 e 46.

54. Si tratta di Purg. XXIII, 85-93 (dove Dante istituirebbe un coperto parallelismo tra Gemma e Nella, sposa di Forese Donati, soprattutto laddove quest'ultimo, vv. 91-93, afferma: «Tanto è a Dio più cara e più diletta / la vedovella mia, che molto amai, / quanto in bene operare è più soletta») e di Par. XVII, 55-57 (dove le parole di Cacciaguida - «tu lascerai ogne cosa diletta / più caramente; e questo è quello strale / che l'arco de lo essilio pria saetta» - dovrebbero riferirsi anche alla moglie del poeta, e non solo ai figli): cfr. per questo G. Sasso, Una postilla per Nella e Gemma Donati, «La cultura», XL, 2002, pp. 443-451, alle pp. 449-451, e Id., A proposito di 'Inferno' XXVI 94-98. Variazioni biografiche per l'interpretazione, ivi, pp. 377-396, alle pp. 378-383.

55. D. Alighieri, Rime, a cura di C. Giunta, in Id., Opere, ed. diretta da M. Santagata, I, Milano, Mondadori, 2011, p. 537; M. Santagata, Le donne di Dante, cit., pp. 39-42.

56. Si tratta dei vv. 81-87: «E se non che degli occhi miei · bel segno / per lontananza m'è tolto dal viso, / che m'have in foco miso, / lieve mi conteria ciò che m'è grave; / ma questo foco m'have / 
sì consumato già l'ossa e la polpa, / che Morte al petto m'ha posto la chiave» (D. Alighieri, Rime, a cura di D. De Robertis, Firenze, Sismel-Edizioni del Galluzzo, 2005, pp. 175-176).

57. La sola altra ricorrenza di gemma all'interno del poema si rintraccia in Par. XV, 22, dove è così definita l'anima di Cacciaguida.

58. D. Alighieri, La Divina Commedia, a cura di U. Bosco e G. Reggio, II (Purgatorio), Firenze, 2002, p. 129.

\section{RIASSUNTI}

Il saggio indaga il rapporto tra biografia e filologia nell'episodio della Pia (Purg. V, 130-136), allo scopo di sottoporre a revisione due luoghi comuni della critica: la 'reticenza' di Dante riguardo alla donna, e la coloritura patetico-elegiaca solitamente attribuita a questi versi. Sottoponendo a nuovo esame il celebre passo nel quadro del canto $\mathrm{V}$ e dell'intero Antipurgatorio, e proponendo la ragionata scelta della lezione disposata al v.136, si giunge a meglio definire la vicenda biografica e il carattere della Pia, nonché a leggere l'episodio in una chiave meno convenzionale e più consona alla tonalità tragica con cui Dante presenta l'esperienza umana dei «morti per forza».

L'article étudie le rapport entre biographie et philologie dans l'épisode de Pia de' Tolomei (Purg. V, 130-136), afin de revisiter deux lieux communs de la critique : la « réticence » de Dante à l'égard de la femme, et la coloration pathétique-élégiaque habituellement attribuée à ces vers. En réexaminant le célèbre passage dans le contexte du chant $\mathrm{V}$ et de l'Anti-purgatoire tout entier, et en proposant le choix raisonné de la leçon exposée au v. 136, on peut mieux définir l'événement biographique et le personnage de Pia, et lire l'épisode de façon moins conventionnelle, plus conforme à la tonalité tragique avec laquelle Dante présente l'expérience humaine des «morts forcés ».

The essay investigates the relationship between biography and philology in the episode of Pia (Purg. V, 130-136), in order to revise two commonplaces of criticism: Dante's 'reticence' regarding the woman, and the pathetic-elegiac tones usually attributed to these verses. Subjecting to a new examination this famous passage in the framework of Canto $\mathrm{V}$ and the entire Antipurgatory, and proposing the reasoned choice of the lesson disposata in v. 136, it is possible to better define the biographical story and the character of Pia, and furthermore, to read the episode in a less conventional key and more in keeping with the tragic tone with which Dante presents the human experience of the "morti per forza".

\section{INDICE}

Mots-clés : Dante, Anti-Purgatoire, philologie, Pia

Parole chiave : Dante, Antipurgatorio, filologia, Pia

Keywords : Dante, Antipurgatory, philology, Pia 


\section{AUTORE}

\section{FRANCESCO BAUSI}

Francesco Bausi è professore ordinario di Letteratura italiana medievale e Filologia italiana presso l'Università della Calabria. Fra le sue ultime pubblicazioni: le edizioni critiche delle Stanze di Angelo Poliziano e del Ciceronianus di Erasmo (2016); il volume Leggere il Decameron (Bologna, 2017); la direzione e la curatela parziale dell'edizione critica delle Lettere di Niccolo' Machiavelli (in corso di stampa).

Francesco Bausi est professeur de littérature italienne du Moyen Âge et de philologie italienne à l'Université de la Calabria. Ses dernières publications comprennent : les éditions critiques des Stanze d'Angelo Poliziano et du Ciceronianus d'Érasme (2016); le volume Leggere il Decameron (Bologne, 2017); la direction et l'édition critique partielle des Lettere de Niccolò Machiavelli (sous presse).

fbausi60@gmail.com 